\title{
A preoperative nomogram predicts prognosis of patients with hepatocellular carcinoma after liver transplantation: a multicenter retrospective study
}

\author{
Dabing Huang ${ }^{1,2,3}$, Yinan Shen ${ }^{1,2,3}$, Wei Zhang ${ }^{1}$, Chengxiang Guo ${ }^{1,2,3}$, Tingbo Liang ${ }^{1,2,3^{*}}$ (D) and Xueli Bai ${ }^{1,2,3^{*}}$
}

\begin{abstract}
Background: Although criteria for liver transplantation, such as the Milan criteria and Hangzhou experiences, have become popular, criteria to guide adjuvant therapy for patients with hepatocellular carcinoma after liver transplantation are lacking.

Methods: We collected data from all consecutive patients from 2012 to 2019 at three liver transplantation centers in China retrospectively. Univariate and multivariate analyses were used to analyze preoperative parameters, such as demographic and clinical data. Using data obtained in our center, calibration curves and the concordance Harrell's $\mathrm{C}$-indices were used to establish the final model. The validation cohort comprised the patients from the other centers.

Results: Data from 233 patients were used to construct the nomogram. The validation cohort comprised 36 patients. Independent predictors of overall survival (OS) were identified as HbeAg positive $(P=0.044)$, blood-type compatibility unmatched $(P=0.034)$, liver transplantation criteria $(P=0.003)$, and high MELD score $(P=0.037)$. For the validation cohort, to predict OS, the C-index of the nomogram was 0.874 . Based on the model, patients could be assigned into low-risk ( $\geq 50 \%)$, intermediate-risk (30-50\%), and high-risk $(\leq 30 \%)$ groups to guide adjuvant therapy after surgery and to facilitate personalized management.
\end{abstract}

Conclusions: The OS in patients with hepatocellular carcinoma after liver transplantation could be accurately predicted using the developed nomogram.

Keywords: Hepatocellular carcinoma, Liver transplantation, Risk factors, Nomogram

\footnotetext{
* Correspondence: liangtingbo@zju.edu.cn; shirleybai@zju.edu.cn 'Department of Hepatobiliary and Pancreatic Surgery, the First Affiliated Hospital, School of Medicine, Zhejiang University, 79 Qingchun Road, Hangzhou 310003, Zhejiang, China

Full list of author information is available at the end of the article
}

(c) The Author(s). 2021 Open Access This article is licensed under a Creative Commons Attribution 4.0 International License, which permits use, sharing, adaptation, distribution and reproduction in any medium or format, as long as you give appropriate credit to the original author(s) and the source, provide a link to the Creative Commons licence, and indicate if changes were made. The images or other third party material in this article are included in the article's Creative Commons licence, unless indicated otherwise in a credit line to the material. If material is not included in the article's Creative Commons licence and your intended use is not permitted by statutory regulation or exceeds the permitted use, you will need to obtain permission directly from the copyright holder. To view a copy of this licence, visit http://creativecommons.org/licenses/by/4.0/. The Creative Commons Public Domain Dedication waiver (http://creativecommons.org/publicdomain/zero/1.0/) applies to the data made available in this article, unless otherwise stated in a credit line to the data. 


\section{Background}

The incidence of liver cancer, despite showing some slow down, increased by 2-3\% annually from 2007 to 2016 [1]. R0 resection and liver transplantation (LT) are regarded as the key methods for the complete remission of liver tumors [2, 3]. LT replaces the diseased liver while simultaneously providing complete oncological resection, which represents a predisposing factor in greater than $90 \%$ of patients with hepatocellular carcinoma (HCC) [4].

Over the last 20 years, various criteria incorporating preoperative variables have been formulated to limit the selection of patients with HCC for LT [5-8]. In 1996, the Milan criteria $(\mathrm{MC})$ were introduced and have been used widely ever since. The MC limited LT to patients with a single tumor with a diameter up to $5 \mathrm{~cm}$ diameter or up to three tumors, none of which is larger than $3 \mathrm{~cm}$ [5]. Twelve years later, the Hangzhou Experience (HZ) was established by our group, which had wider surgical indications than the $\mathrm{MC}$ and was more suitable for Asian populations [6]. Thereafter, criteria for postoperative guidance were lacking until Agopian et al. reported a prognostic system that could accurately predict HCC recurrence after LT [4]. Unfortunately, this model focused on HCC recurrence and was constructed on Caucasian population data. Therefore, we established this large, multiple-center retrospective study comprising Chinese patients with HCC who underwent LT at three liver transplantation centers in China. The study aimed to formulate a nomogram to predict the long-term survival of these patients, to guide adjuvant therapy, and facilitate personalized management.

\section{Methods}

\section{Patients participating in the study}

The present study included patients who were diagnosed with HCC and underwent LT from 2012 to 2019 at three liver transplantation centers in China. The three centers comprised: the First Affiliated Hospital of Zhejiang University School of Medicine in Hangzhou, Tianjin First Center Hospital in Tianjin, and the Third Affiliated Hospital of Sun Yat-sen University in Guangzhou. These institutions perform high volumes of liver transplantation. We only included patients who underwent LT and who had histopathologically confirmed HCC. The inclusion criteria comprised: no history of other malignant tumors; and no surgical procedures other than LT. If a patient died within 30 days of LT, they were excluded from this study.

\section{Imaging and pathological analysis}

Magnetic resonance imaging (MRI) or computed tomography $(\mathrm{CT})$ were used to determine the extent of pretransplantation disease. A radiologist who was blinded to the patients' clinical records analyzed all the images retrospectively. The tumors of patients with HCC were classified as within the Milan criteria, beyond the Milan criteria but the within Hangzhou criteria, or exceeding the Hangzhou criteria. All liver explants were reviewed by an experienced pathologist and categorized based on lymph node involvement, presence of macro- and microvascular invasion, histological grade and differentiation, distribution, size, and tumor number. The tumor-nodemetastasis (TNM) stage of the patients with HCC in our cohort was based on the America Joint Committee on Cancer TNM staging system (8th edition) [9].

\section{Patient follow-up}

The patients were subjected to follow-up once every 2 weeks in the first 3 months, every 4 weeks in the first 1 year, and then once every 3 months. A detailed clinical history was recorded and a complete physical examination was performed at each follow-up visit. This study was censored on Dec. 31st, 2019.

\section{Statistical analysis}

SPSS 19.0 for Windows (IBM Corp., Armonk, NY, USA) and Prism 6 for Windows version 6.01 (GraphPad Software, San Diego, CA, USA) were used to identify risk factors. Clinical findings were used to group the categorical variables, and the groups were decided upon before modeling. The chi-squared test or Fisher's exact test were used to compare all the results. To compare continuous variables, we used Student's t-test or the Mann-Whitney U test for variables with abnormal distributions. The log-rank test was used to compare survival curves that had been constructed using the KaplanMeier method. Multivariate analysis was executed using Cox regression analysis.

The results of multivariate analysis formed the basis to construct the nomogram, using the rms26 package in $\mathrm{R}$ version 3.3.2 (http://www.r-project.org/). A backward step-down selection process with the Akaike information criterion was used to perform the final model selection [10]. The concordance index (C-index) was used to measure the performance of the nomogram. A comparison of the nomogram-predicted versus the observed Kaplan-Meier estimates of survival probability formed the basis of the assessment of nomogram performance. During nomogram validation, the established nomogram was used to calculate the total points for each patient from the validation cohort. Subsequently, the total points were used as a factor in the Cox regression analysis in this cohort. Finally, the regression analysis was used to derive the $\mathrm{C}$-indices and the calibration curves. Statistical significance was accepted at a $P$ value less than 0.05 . 


\section{Results}

\section{Characteristics of the patients}

Three liver transplantation centers provided retrospective data for 281 patients. Twelve patients in our center died within 30 days after surgery, including 7 cases of postoperative acute immune rejection, 3 cases of massive postoperative bleeding, and 2 cases of severe lung infection. Other patients in our center were used as the training cohort $(n=233)$ and the patients in the remaining centers comprised the validation cohort $(n=36)$. There was a near 6:1 proportion of patients between two groups. The patients' baseline characteristics are shown in Table 1.

\section{Overall survival (OS)-related prognostic factors}

Univariate and multivariate Cox regression analyses were used to assess the demographic, clinical, and biochemical parameters of the training cohort to identify independent risk factors associated with OS. The results revealed that OS was significantly associated with the presence of ascites, HbeAg positive, blood-type compatibility unmatched, liver transplantation criteria, and high model for end-stage liver disease (MELD) (Table 2). Upon multivariate analysis, HbeAg positive, blood-type compatibility unmatched, liver transplantation criteria, and a high MELD score were suggested as independent OS-related risk factors (Table 2).

\section{OS-associated risk factors-based prognostic nomogram}

The four identified independent risk factors for OS were used to construct a nomogram to predict the survival of patients with HCC after LT (Fig. 1). The odds ratio (OR) was used to weight the assignment of points to each factor. The total score was then used to calculate the survival probability at specific times (1, 2 and 3 years postLT). For example, a patient with HCC after LT within the MC criteria (0 point), with a MELD score of 20 (50 points), HbeAg positive (26 points), and blood-type compatibility matched (0 points) would have a total score of 76 points, which corresponded to survival probabilities of 79,58 , and $51 \%$ at 1,2 , and 3 years, respectively.

Using the nomogram, the training cohort received a C-index for the prediction of OS of 0.677 (95\% CI, 0.606-0.748; Fig. 2, upper panel), and the C-index was 0.874 for the validation cohort (95\% CI, 0.753-0.995; Fig. 2, lower panel).

\section{Risk groups}

Three risk groups were identified using the risk scores calculated using the nomogram (Table 3): 1) The lowrisk group (total points $\leq 79$ and a predicted survival rate $\geq 50 \%$ ), with a mean predicted mean survival rate $=$ $68.2 \%$; 2) The intermediate-risk group (total points 79-
107 and a predicted survival rate of $30-50 \%$ ), with a mean predicted survival rate $=39.6 \%$; and 3 ) the highrisk group (total points $\geq 107$ and a predicted survival rate $\leq 30 \%$ ), with a mean predicted survival rate $=20.1 \%$.

The observed survival rate conformed completely with the mean predicted survival rate. Among the risk groups there were significant differences in survival $(P<0.0001)$. Figure 3 shows the cumulative patient survival rates for the three groups. The low risk group had significantly better survival than the intermediate-risk group $(P=$ $0.000)$. There was also a significant difference between the high-risk and intermediate-risk groups $(P=0.009)$.

\section{Discussion}

Whether LT is the best treatment option for $\mathrm{HCC}$ is a matter of debate [6]. However, currently, LT is widely accepted as the best treatment modality. The introduction of the Milan criteria (MC) represented a breakthrough in the standardization of LT and the Hangzhou experiences (HZ) further broadened the indications for surgery. However, increasing evidence showed that these criteria are too conservative and only focus on the tumor status. In fact, whole body condition of patients and other parameters, such as liver function, radiological appearance, and epidemiological factors, can also affect the outcome of LT. The present study aimed to identify independent risk factors for prognosis in patients with HCC who received LT. These risk factors were then used to establish a nomogram for the presurgical prediction of OS.

During the construction of the nomogram, we did not try to replace the original standards, such as the MC or $\mathrm{HZ}$ criteria. We included these criteria and other parameters, including laboratory results, epidemiological factors, and the previous history of the patients, but not tumor status. As expected, multivariate analysis identified the LT criteria as one of the risk factors of prognosis. Patients with tumors within the $\mathrm{MC}$ criteria, beyond the $\mathrm{MC}$ but within the $\mathrm{HZ}$ criteria, or exceeding the $\mathrm{HZ}$ criteria were given different statistical weights. Patients with tumors exceeding the $\mathrm{HZ}$ criteria received 45 points and had the worst prognosis because of advanced tumor and poor patient status. Initially, the MELD score was designed to evaluate renal and hepatic dysfunctioninduced organ failure. However, it has been wellvalidated to guide organ allocation policy and to identify patients who are at risk of death because of end-stage liver disease [11]. In general, organs with a high donor risk index (DRI) should be transplanted into patients with a low MELD score of 10-14 [12, 13]. However, subsequent data showed that patients with high MELD scores could also benefit from sub-optimal organ transplants [12-14]; therefore, we included the entire range of MELD scores into our model. 
Table 1 Patient Baseline Characteristics by Cohort

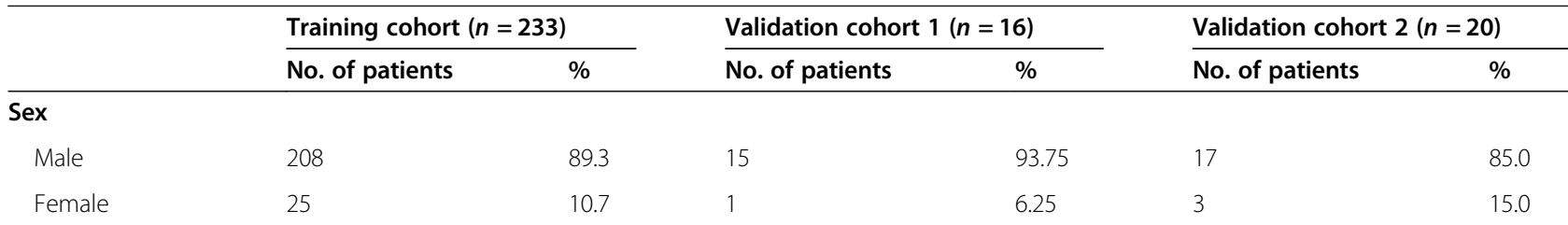

Age, years

$\begin{array}{llll}\text { Median } & 53.0 & 53.0 & 53.5 \\ \text { IQR } & 48.0-60.0 & 47.3-61.8 & 47.8-62.0\end{array}$

\section{LT criteria}

\begin{tabular}{|c|c|c|c|c|c|c|}
\hline Milan & 95 & 40.8 & 14 & 87.5 & 14 & 70.0 \\
\hline Hangzhou & 42 & 18.0 & 2 & 12.5 & 4 & 20.0 \\
\hline Over Hangzhou & 96 & 41.2 & 0 & 0 & 2 & 10.0 \\
\hline \multicolumn{7}{|l|}{ Smoke } \\
\hline Yes & 128 & 54.9 & 13 & 81.2 & 12 & 60.0 \\
\hline No & 105 & 45.1 & 3 & 18.8 & 8 & 40.0 \\
\hline \multicolumn{7}{|l|}{ Alcohol } \\
\hline Yes & 77 & 33.0 & 10 & 62.5 & 8 & 40.0 \\
\hline No & 156 & 67.0 & 6 & 37.5 & 12 & 60.0 \\
\hline
\end{tabular}

BMI

Median

IQR

22.8

21.1-25.1

AFP, $\mathrm{ng} / \mathrm{mL}$

$\begin{array}{ll}\text { Median } & 13.9 \\ \text { IQR } & 3.4-256.8\end{array}$

\section{CEA, ng/ml}

Median

2.6

IQR

$1.8-4.1$

TB, $\mu \mathrm{mol} / \mathrm{mL}$

$\begin{array}{ll}\text { Median } & 26.0 \\ \text { IQR } & 15-55.5\end{array}$

Albumin, g/L

Median

$$
34.0
$$

30.4-38.3

ALT, U/L

Median

IQR

34.0

$20.5-60.5$

AST, U/L

$\begin{array}{ll}\text { Median } & 45.0 \\ \text { IQR } & 31-80\end{array}$

Hypertension

\begin{tabular}{|c|c|c|}
\hline Yes & 40 & 17.2 \\
\hline No & 193 & 82.8 \\
\hline
\end{tabular}

Diabetes

$\begin{array}{ll}\text { Yes } & 26 \\ \text { NO } & 207\end{array}$

\section{9}

21.2-25.6

21.5

19-22.9

31.1

6.2-426.1

174.6

6.9-2915.2

1.3

$1.0-1.9$

2.8

$1.8-3.8$

25.1

10.4-120.8

26.1

15.7-76.6

$\begin{array}{ll}37.9 & 35.8 \\ 34.5-40.7 & 18.3-44.1\end{array}$

33.5

21.5-62.8

35.8

$18.3-44.1$

32.0

$24.3-110.3$

47.6

$26.2-74.1$ 
Table 1 Patient Baseline Characteristics by Cohort (Continued)

\begin{tabular}{|c|c|c|c|c|c|c|}
\hline & \multicolumn{2}{|c|}{ Training cohort $(n=233)$} & \multicolumn{2}{|c|}{ Validation cohort $1(n=16)$} & \multicolumn{2}{|c|}{ Validation cohort $2(n=20)$} \\
\hline & No. of patients & $\%$ & No. of patients & $\%$ & No. of patients & $\%$ \\
\hline \multicolumn{7}{|l|}{ Ascites } \\
\hline No & 142 & 60.9 & 9 & 56.3 & 13 & 65.0 \\
\hline Mild & 70 & 30.0 & 6 & 37.5 & 1 & 5.0 \\
\hline Severe & 21 & 9.1 & 1 & 6.3 & 6 & 30.0 \\
\hline \multicolumn{7}{|l|}{$\mathrm{HBeAg}$} \\
\hline Positive & 56 & 24.0 & 15 & 93.8 & 17 & 85.0 \\
\hline Negative & 177 & 76.0 & 1 & 6.2 & 3 & 15.0 \\
\hline \multicolumn{7}{|l|}{ Blood type } \\
\hline Matched & 189 & 81.1 & 15 & 93.8 & 19 & 95.0 \\
\hline Unmatched & 44 & 18.9 & 1 & 6.2 & 1 & 5.0 \\
\hline
\end{tabular}

Table 2 Univariate and multivariate analyses of factors associated with overall survival

\begin{tabular}{|c|c|c|c|c|c|c|}
\hline & \multicolumn{6}{|c|}{ Overall survival } \\
\hline & \multicolumn{3}{|c|}{ Univariate analysis } & \multicolumn{3}{|c|}{ Multivariate analysis } \\
\hline & $\mathrm{HR}$ & $95 \% \mathrm{Cl}$ & $P$ value & $\mathrm{HR}$ & $95 \% \mathrm{Cl}$ & $P$ value \\
\hline Age, years & 1.009 & $0.984-1.036$ & 0.475 & & & \\
\hline Sex (male/female) & 1.120 & $0.515-2.439$ & 0.775 & & & \\
\hline Smoking (yes/no) & 1.266 & $0.801-2.000$ & 0.313 & & & \\
\hline Drinking (yes/no) & 1.472 & $0.926-2.342$ & 0.102 & & & \\
\hline $\mathrm{BMI}, \mathrm{kg} / \mathrm{m}^{2}$ & 0.999 & $0.995-1.003$ & 0.613 & & & \\
\hline Hypertension (absence/ presence) & 1.211 & $0.665-2.204$ & 0.531 & & & \\
\hline Diabetes (absence/ presence) & 1.339 & $0.687-2.610$ & 0.391 & & & \\
\hline Ascites (absence/ presence) & 1.473 & $1.079-2.011$ & 0.015 & 1.132 & $0.802-1.598$ & 0.480 \\
\hline HbsAg (negative/positive) & 1.535 & $0.667-3.534$ & 0.314 & & & \\
\hline HbeAg (negative/positive) & 1.696 & $1.056-2.725$ & 0.029 & 1.640 & $1.014-2.651$ & 0.044 \\
\hline Blood-type compatibility (matched/unmatched) & 2.252 & $1.378-3681$ & 0.001 & 1.733 & $1.034-2.880$ & 0.034 \\
\hline Liver transplantation criteria & 1.678 & $1.279-2.203$ & 0.000 & 1.536 & $1.157-2.037$ & 0.003 \\
\hline MELD score & 1.058 & $1.019-1.100$ & 0.004 & 1.044 & $1.003-1.088$ & 0.037 \\
\hline Child-Pugh classification & 1.001 & $0.895-1.121$ & 0.980 & & & \\
\hline WBC, $10^{*} 9 / \mathrm{L}$ & 1.036 & $0.996-1.077$ & 0.078 & & & \\
\hline$H G B, g / L$ & 1.004 & $0.996-1.013$ & 0.313 & & & \\
\hline PLT, $10^{*} 9 / \mathrm{L}$ & 1.002 & $0.999-1.005$ & 0.112 & & & \\
\hline INR & 0.574 & $0.295-1.119$ & 0.103 & & & \\
\hline Serum CRE, $\mu \mathrm{mol} / \mathrm{L}$ & 0.998 & $0.994-1.002$ & 0.343 & & & \\
\hline Serum AFP, ng/mL & 1.000 & $1.000-1.000$ & 0.082 & & & \\
\hline Serum CEA, ng/mL & 0.944 & $0.850-1.049$ & 0.283 & & & \\
\hline Serum CA125, U/L & 1.000 & $0.999-1.001$ & 0.613 & & & \\
\hline Serum ALT, U/L & 1.001 & $0.999-1.003$ & 0.279 & & & \\
\hline Serum AST, U/L & 1.000 & $1.000-1.000$ & 0.416 & & & \\
\hline Serum ALP, U/L & 0.999 & $0.997-1.001$ & 0.190 & & & \\
\hline Serum GGT, U/L & 0.999 & $0.997-1.001$ & 0.114 & & & \\
\hline Serum TB, $\mu \mathrm{mol} / \mathrm{L}$ & 0.999 & $0.997-1.002$ & 0.654 & & & \\
\hline Serum ALB, g/L & 1.005 & $0.966-1.046$ & 0.795 & & & \\
\hline
\end{tabular}

Cl confidence interval, HR hazard ratio, BMI body mass index, MELD model for end-stage liver disease, WBC white blood cell, HGB hemoglobin, PLT platelets, INR international normalized ratio, CRE creatinine, AFP alpha fetoprotein, CEA carcino-embryonic antigen, CA125 carbohydrate antigen 125, ALT alanine aminotransferase, AST aspartate aminotransferase, ALP alkaline phosphatase, GGT gamma-glutamyl transpeptidase, $T B$ total bilirubin, $A L B$ albumin 


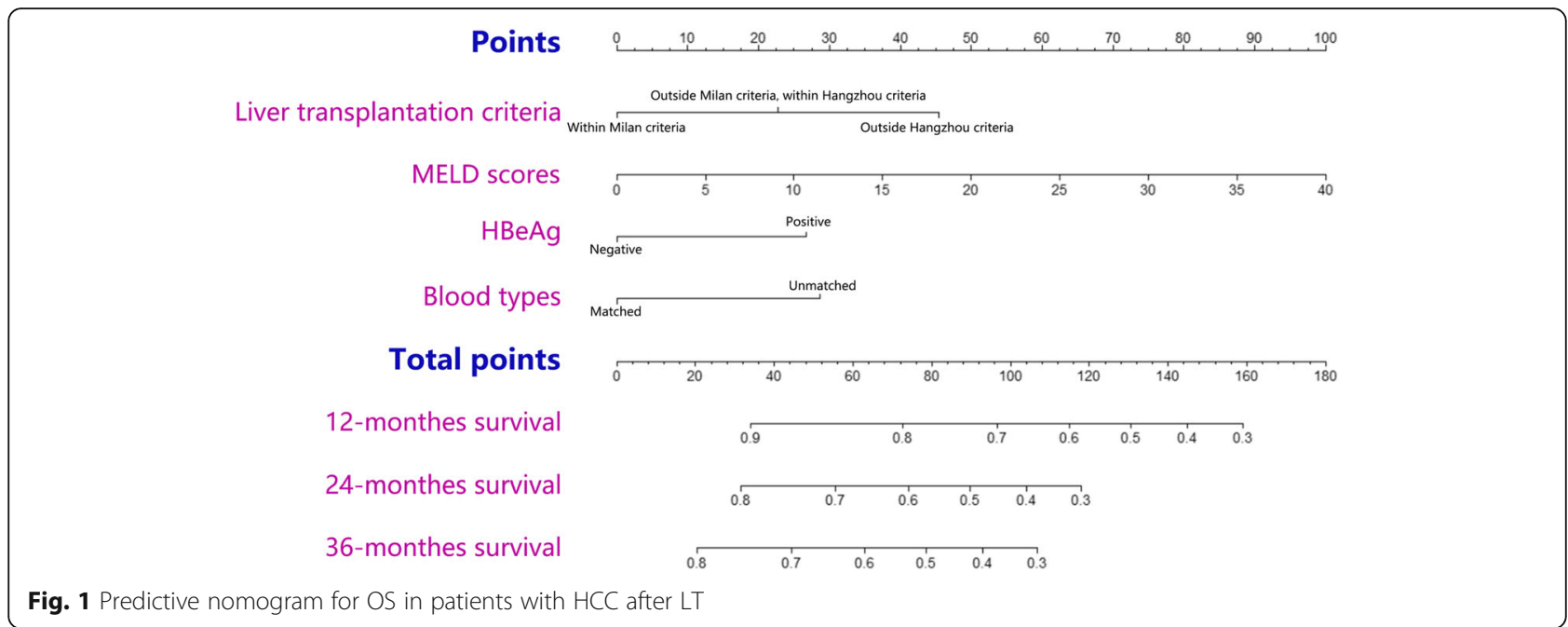

In this study, HbeAg positive was found a risk factor of OS. It was reported that the risk of HCC increased significantly when associated with a single positive HbeAg [15]. Other studies reported that persistent viremia might result in active liver disease after $\mathrm{HbeAg}$ seroconversion or fluctuating $\mathrm{HBeAg}$ status, which would increase the risk of HCC and liver cirrhosis in these patients [16, 17]. However, Chan et al. reported that compared with HBeAg positivity, HBV-DNA is a more important risk factor for HCC [18]. Unfortunately, the data on HBV-DNA was lacking in our study, which might have affected the accuracy of our nomogram. In addition, HbeAg positivity always occurs in the active phase of hepatitis. Most HCC are due to cirrhosis caused by $\mathrm{HBeAg}$ positive, and cirrhosis is the most important risk factor for HCC. Studies on the correlation between cirrhosis and HCC have shown that the forming procession of HCC is largely mediated by inflammation, which causes the cycle facts of cell death and regeneration and induces the repeated proliferation of liver cells

\section{A}

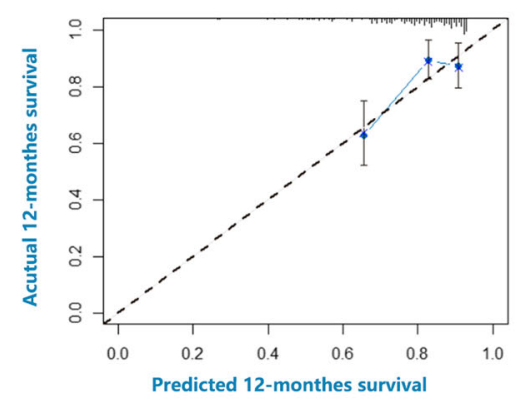

D

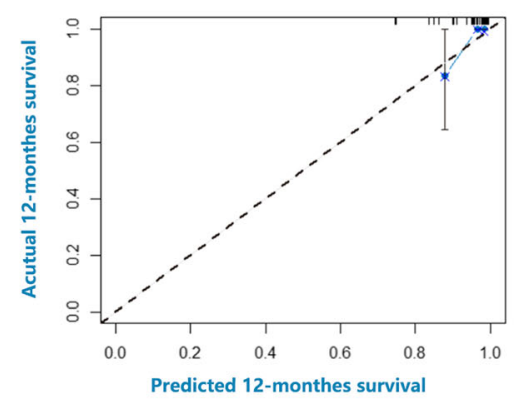

B

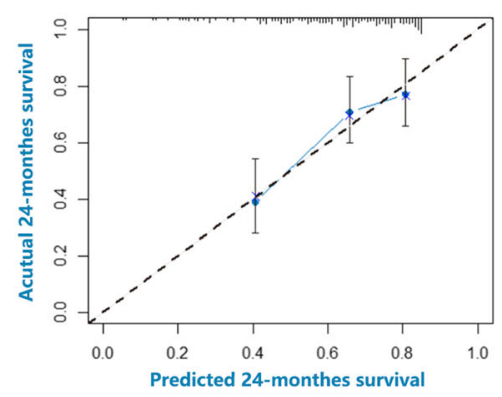

E

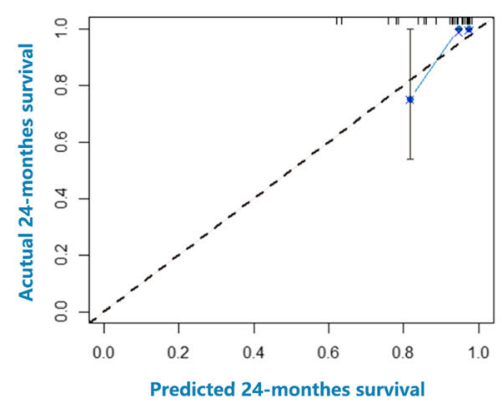

C

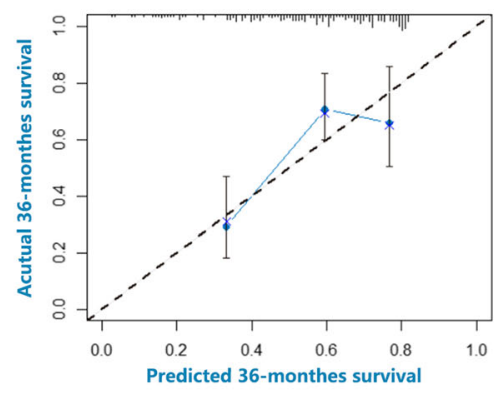

F

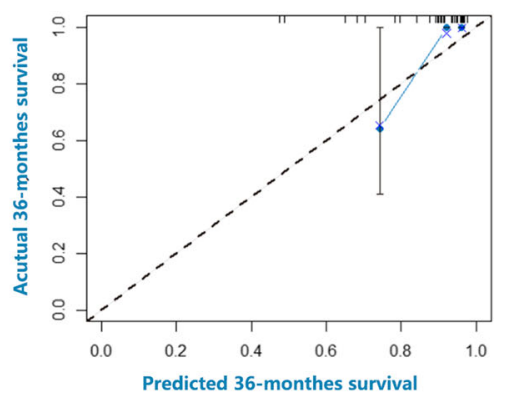

Fig. 2 Calibration curves for predicting patients' survival at (a) 12 months, (b) 24 months, and (c) 36 months in the training cohort and at (d) 12 months, (e) 24 months, (f) 36 months in the validation cohort 
Table 3 Risk groups based on the predicted nomogram

\begin{tabular}{lllll}
\hline Group & Total Points & Predicted Survival Rate & Predicted Mean Survival Rate & Observed Survival Rate \\
\hline High-risk group & $>=107$ & $<=30 \%$ & $20.1 \%$ & $28.0 \%(7 / 25)$ \\
Intermediate-risk group & $79-107$ & $30-50 \%$ & $39.6 \%$ & $48.1 \%(25 / 52)$ \\
Low-risk group & $<=79$ & $>=50 \%$ & $68.2 \%$ & $78.2 \%(122 / 156)$ \\
\hline
\end{tabular}

[19]. The release of inflammatory factors and poor patient status will also affect the result of LT. Hepatitis B $\mathrm{X}$ protein $(\mathrm{HBx})$ is encoded by $\mathrm{HBV}$, and it has been demonstrated that $\mathrm{HBx}$ is closely linked to HBV-related HCC [20]. HBx plays a multifunctional role in common biochemical pathways by modulating the expression and activities of numerous genes, contributing to many pathological processes, including viral replication, gene transcription, signal transduction and protein degradation, which are ultimately linked to the occurrence and development of HCC [21, 22].

Studies have shown increased prevalence of antibodymediated rejection and cholangitis, lower graft survival and hepatic artery thrombosis in ABO- unmatched LT compared with those in ABO-matched LT. These observations could explain the results of the present study. In addition, a meta- analysis also showed worse graft survival in $\mathrm{ABO}$ - unmatched $\mathrm{LT}$ compared with that in ABO- matched LT [23]. When the blood type of A, B and $\mathrm{O}$ are incompatible, the antibody of anti- $\mathrm{A}$ or $\mathrm{B}$ in recipient's blood will combine with the antigen directly, which is on the vascular endothelial cells of graft, and produce an antigen-antibody complex, activate the complement system quickly. As a result, it will destroy the vascular plexus and small bile ducts in the graft, form the extensive thrombotic microangiopathy, cause a series of rejection reactions and complications. The above factors will reduce the survival rate of the graft [24].
This study has some limitations. First, our study included four clinical indicators and no biological ones. Second, despite having 269 patients in this study, the validation cohort had a relatively small sample size, which might have resulted in its high C-index (0.874). Similarly, due to the limited samples size in the high-risk group and there are only two long-term survivors after 20 months, it may cause our nomogram to have a certain deviation in the assessment of 24 months and 36 months. Larger-sized cohorts are required to further determine the clinical value of the nomogram. In addition, the $\mathrm{C}$ index will become more accurate with the expansion of the sample size. Second, as stated above, the lack of HBV-DNA data might have influenced the application value of our model, and we hope to fix this problem in future.

\section{Conclusion}

A novel nomogram for OS prediction of patients with HCC after LT was constructed based on four essential independent risk factors determined at diagnosis. This nomogram showed good performance, with a Cindex $=0.874$ for the external validation cohort. The model allowed us to classify patients with HCC after LT into three risk groups. The identified risks could be used to provide specific management strategies in the future.

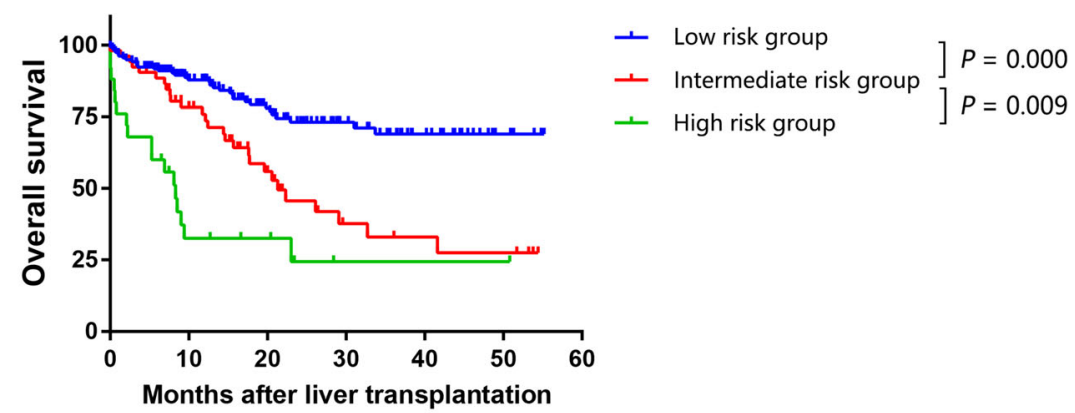

No. at risk

$\begin{array}{lccccccc}\text { Low-risk group } & 156 & 106 & 68 & 39 & 23 & 7 & 0 \\ \text { Inter-risk group } & 52 & 37 & 21 & 9 & 7 & 6 & 0 \\ \text { High-risk group } & 25 & 8 & 6 & 2 & 2 & 2 & 0\end{array}$

Fig. 3 Cumulative survival rates in the different risk groups (the training cohort) 


\section{Abbreviations}

LT: Liver transplantation; HCC: Hepatocellular carcinoma; MC: Milan criteria (MC); HZ: Hangzhou Experience; MRI: Magnetic resonance imaging; CT: Computed tomography; TNM: The tumor-node-metastasis; OS: Overall survival; MELD: Model for end-stage liver disease; OR: Odds ratio

\section{Acknowledgements}

We thank Organ Transplant Center of Tianjin First Center Hospital, and Liver Transplantation Center of the Third Affiliated Hospital of Sun Yat-sen University for providing their data.

\section{Authors' contributions}

HDB, SYN, ZW contributed equally to this work. HDB, LTB, BXL, SYN, and ZW conceived and designed this study. The manuscript was written by HDB and SYN. HDB provide the study materials and collect the data. HDB, SYN, ZW, and GCX performed the statistical analyses and interpreted the results of data. All authors read and approved the final manuscript.

\section{Funding}

This study was funded by the grants from the National Key Research and Development Program (grant number 2019YFC1316000), the National Natural Science Foundation of China (grant numbers 31970696, 81502975 and 81802355), and the Zhejiang Provincial Program for the Cultivation of High-level Innovative Health Talents. The funders played no roles in the study design, the collection, analysis, and interpretation of data, the writing of the report, and the decision to submit the article for publication. All authors, external and internal, had full access to all of the data (including statistical reports and tables) in the study and can take responsibility for the integrity of the data and the accuracy of the data analysis, as well as being independent of the funders.

\section{Availability of data and materials}

The datasets used and analysed during the current study available from the corresponding author on reasonable request.

\section{Ethics approval and consent to participate}

The Ethics Committees of the First Affiliated Hospital (the First Affiliated Hospital, School of Medicine, Zhejiang University) have approved this study. All patients provided wWritten informed consent for their data to be used for research was provided by all patients before LT for their data to be used for research. All liver explants were donated by volunteers who signed donation agreements while living.

\section{Consent for publication}

Not applicable.

\section{Competing interests}

All of authors declare that they have no competing interests.

\section{Author details}

'Department of Hepatobiliary and Pancreatic Surgery, the First Affiliated Hospital, School of Medicine, Zhejiang University, 79 Qingchun Road, Hangzhou 310003, Zhejiang, China. ${ }^{2}$ Zhejiang Provincial Key Laboratory of Pancreatic Disease, the First Affiliated Hospital, School of Medicine, Zhejiang University, Hangzhou 310003, Zhejiang, China. ${ }^{3}$ Zhejiang Provincial Innovation Center for the Study of Pancreatic Diseases, Hangzhou 310003, Zhejiang, China.

Received: 3 June 2020 Accepted: 19 February 2021 Published online: 16 March 2021

\section{References}

1. Siegel RL, Miller KD, Jemal A. Cancer statistics, 2020. CA Cancer J Clin. 2020; 70(1):7-30.

2. Shen $Y N$, Guo $C X$, Wang $L Y$, et al. Associating liver partition and portal vein ligation versus 2-stage hepatectomy: a meta-analysis. Medicine (Baltimore). 2018;97(35):e12082.

3. Rahbari NN, Mehrabi A, Mollberg NM, et al. Hepatocellular carcinoma: current management and perspectives for the future. Ann Surg. 2011;253(3):453-69.

4. Agopian VG, Harlander-Locke M, Zarrinpar A, et al. A novel prognostic nomogram accurately predicts hepatocellular carcinoma recurrence after liver transplantation: analysis of 865 consecutive liver transplant recipients. J Am Coll Surg. 2015;220(4):416-27.

5. Mazzaferro V, Regalia E, Doci R, et al. Liver transplantation for the treatment of small hepatocellular carcinomas in patients with cirrhosis. N Engl J Med. 1996;334(11):693-9.

6. Zheng SS, Xu X, Wu J, et al. Liver transplantation for hepatocellular carcinoma: Hangzhou experiences. Transplantation. 2008;85(12):1726-32.

7. Yao FY, Ferrell L, Bass NM, et al. Liver transplantation for hepatocellular carcinoma: expansion of the tumor size limits does not adversely impact survival. Hepatology. 2001;33(6):1394-403.

8. Mazzaferro V, Llovet JM, Miceli R, et al. Predicting survival after liver transplantation in patients with hepatocellular carcinoma beyond the Milan criteria: a retrospective, exploratory analysis. Lancet Oncol. 2009;10(1):35-43.

9. Allen PJ, Kuk D, Castillo CF, et al. Multi-institutional validation study of the American joint commission on Cancer (8th edition) changes for $\mathrm{T}$ and $\mathrm{N}$ staging in patients with pancreatic adenocarcinoma. Ann Surg. 2017;265(1):185-91.

10. Harrell FE, Lee KL, Mark DB. Multivariable prognostic models: issues in developing models, evaluating assumptions and adequacy, and measuring and reducing errors. Stat Med. 1996;15(4):361-87.

11. Wiesner R, Edwards E, Freeman R, et al. Model for end-stage liver disease (MELD) and allocation of donor livers. Gastroenterology. 2003;124(1):91-6.

12. Bonney GK, Aldersley MA, Asthana S, et al. Donor risk index and MELD interactions in predicting long-term graft survival: a single-Centre experience. Transplantation. 2009;87(12):1858-63.

13. Maluf DG, Edwards EB, Kauffman HM. Utilization of extended donor criteria liver allograft: is the elevated risk of failure independent of the model for endstage liver disease score of the recipient. Transplantation. 2006;82(12):1653-7.

14. Rauchfuss F, Zidan A, Scheuerlein $H$, et al. Waiting time, not donor-riskindex, is a major determinant for beneficial outcome after liver transplantation in high-MELD patients. Ann Transplant. 2013;18:243-7.

15. Geier A, Gartung C, Dietrich CG. Hepatitis B e antigen and the risk of hepatocellular carcinoma. N Engl J Med. 2002;347(21):1721-2 author reply 1721-2.

16. Di MV, Lo IO, Cammà C, et al. The long-term course of chronic hepatitis B. Hepatology. 1999;30(1):257-64.

17. McMahon BJ, Holck P, Bulkow L, et al. Serologic and clinical outcomes of 1536 Alaska natives chronically infected with hepatitis B virus. Ann Intern Med. 2001;135(9):759-68.

18. Chan HL, Tse CH, Mo F, et al. High viral load and hepatitis B virus subgenotype ce are associated with increased risk of hepatocellular carcinoma. J Clin Oncol. 2008;26(2):177-82.

19. Shin HR, Lee CU, Park HJ, et al. Hepatitis B and C virus, Clonorchis sinensis for the risk of liver cancer: a case-control study in Pusan, Korea. Int J Epidemiol. 1996;25(5):933-40.

20. Ali A, Abdel-Hafiz H, Suhail M, et al. Hepatitis B virus, HBx mutants and their role in hepatocellular carcinoma. World J Gastroenterol. 2014;20(30):10238-48.

21. Zhu K, Huang W, Wang W, et al. Up-regulation of S100A4 expression by HBx protein promotes proliferation of hepatocellular carcinoma cells and its correlation with clinical survival. Gene. 2020;749:144679.

22. Liu S, Koh SS, Lee CG. Hepatitis B Virus X Protein and Hepatocarcinogenesis. Int J Mol Sci. 2016:17(6):940-54.

23. Lee EC, Kim SH, Park SJ. Outcomes after liver transplantation in accordance with $\mathrm{ABO}$ compatibility: a systematic review and meta-analysis. World J Gastroenterol. 2017;23(35):6516-33.

24. Kishida N, Shinoda M, Itano O, et al. Increased Incidence of Thrombotic Microangiopathy After ABO-Incompatible Living Donor Liver Transplantation. Ann Transplant. 2016;21:755-64

\section{Publisher's Note}

Springer Nature remains neutral with regard to jurisdictional claims in published maps and institutional affiliations. 